\title{
Incidence and Management Trends of Osteoporotic Vertebral Compression Fractures in South Korea: A Nationwide Population-Based Study
}

\author{
Sung Hoon Choi, Dong-Yun Kim, Ja Wook Koo, Seung Gun Lee, Soo-Young Jeong, Chang-Nam Kang \\ Department of Orthopedic Surgery, Hanyang University College of Medicine, Seoul, Korea
}

\section{Study Design: A nationwide population-based study.}

Purpose: Osteoporotic vertebral compression fracture (OVCF) is a major public health issue. This study examined the incidence and management trends of OVCF in South Korea.

Overview of Literature: The incidence rates, management trends, and patterns of OVCF differ in different parts of the world. The age-standardized OVCF incidence rate in 2015 was higher in the United States and Asia than in Europe.

Methods: A nationwide database (2012-2016) acquired from the Korean Health Insurance Review and Assessment Service was analyzed. International disease categories in the 10th revision of the International Statistical Classification of Diseases and Related Health Problems codes, medical procedure codes, and examination codes were used to identify and sort OVCF patients aged $>50$ years.

Results: There were 644,500 OVCF cases from 2012 to 2016. OVCF was most common in patients in their seventies (45\%) and the number of patients increased from 117,361 in 2012 to 139,889 in $2016(p<0.001)$. During 2012-2016, 8.9\% of patients visited the emergency department; of those, $54.3 \%$ were hospitalized and $35 \%$ underwent magnetic resonance imaging. In OVCF treatment, bone cement augmentation rates increased from $23.4 \%$ in 2012 to $25.2 \%$ in 2016 ( $p<0.001)$, while conservative treatment rates slightly decreased from $76.5 \%$ in 2012 to $74.7 \%$ in 2016 ( $p<0.001)$. The total health insurance cost was $\$ 193,210,353.55$ in 2012 and $\$ 281,968,877.65$ in 2016 .

Conclusions: The 5-year incidence of OVCF per 100,000 persons was 852.24 cases, and 45\% of OVCF in South Korea occurred in patients in their seventies. The bone cement augmentation rate and total cost of OVCF are continuously increasing.

Keywords: Thoracolumbar spine; Senile osteoporosis; Compression fractures; Trends; Republic of Korea

\section{Introduction}

Osteoporosis is characterized by bone microarchitecture destruction and bone quality degradation [1], leading to decreased bone strength due to decreased bone mineral density (BMD) or poor bone quality. Osteoporotic frac- tures can occur easily with minor trauma or even without any noticeable trauma in these patients [2]. As the morbidity and mortality rates of osteoporotic fractures are continuously rising, these fractures have gained increasing attention. Osteoporotic vertebral compression fracture (OVCF) is the most common type of osteoporotic fracture

Received Feb 14, 2019; Revised May 22, 2019; Accepted May 27, 2019

Corresponding author: Chang-Nam Kang

Department of Orthopaedic Surgery, Hanyang University College of Medicine, 222-1 Wangsimni-ro, Seongdong-gu, Seoul 04763, Korea Tel: +82-2-2290-8485, Fax: +82-2-2290-3774, E-mail: cnkang65@hanyang.ac.kr 
worldwide, and its prevalence is gradually increasing with the aging population. OVCF affects the quality of life because of the constant pain, and studies have suggested that it could occur in $30 \%-50 \%$ of the global population over $50[2,3]$.

The prevalence of OVCF has been studied in various countries. Sakuma et al. [4] in 2014 reported an OVCF incidence rate of 653 per 100,000 persons, while the European Prospective Osteoporosis Study Group reported an OVCF incidence rate of 570 per 100,000 persons for men and 1,070 per 100,000 persons for women [5]. A global OVCF study (2017) reported that the age-standardized OVCF incidence rate in 2015 was higher in the United States and Asia (South Korea, Hong Kong) compared to Europe [3].

In addition to prevalence, the social costs of OVCF have also been studied. According to the US data for 2005 published by Burge et al. [6], the total cost of treating 2.05 million osteoporotic fracture cases was $\$ 16.9$ billion. Of these osteoporotic fractures, OVCF was the second-most common fracture (27\%), and its cost was $\$ 1$ billion, or $6 \%$ of the $\$ 16.9$ billion. Researchers anticipate that by 2025 , the OVCF incidence rate will increase by $52 \%$ and its cost by $53 \%$. An Austrian study conducted from August 1990 to January 2012 reported that in the on 99,399 women studied, there were 6,540 osteoporosis fracture cases, of which 3,070 (47\%) had fractures other than those of the vertebrae (distal forearm and pelvic fractures) [7]. Unlike distal forearm fractures, pelvic fractures and vertebral compression fractures are found to increase mortality rates [8].

OVCF incidence rates, management trends, and patterns OVCF differ in different of the world. Although several studies have used nationwide registers or records, they are based on Western countries. In addition, some studies have several limitations, for example, use of hospitalization-based information. Therefore, this study aimed to investigate the incidence rates and management trends of OVCF in South Korean populations aged $>50$ years using a nationwide database.

\section{Materials and Methods}

\section{Data source and collection}

All clinics and hospitals report data on inpatients and outpatients, including diagnosis, treatment, and medical costs, for claims to the Korean Health Insurance Review and Assessment Service (HIRA). Therefore, all information about patients and diseases is available from HIRA database, which is often used for epidemiological studies in South Korea. Our study cohort was also identified from the nationwide National Korean Health Insurance claims database for 5 years (2012-2016) registered in HIRA. Patients aged $>50$ years diagnosed with OVCF were selected and sorted using the medical procedure and examination codes of 10th revision of the International Statistical Classification of Diseases and Related Health Problems (ICD10) (Table 1). After determining the age and sex of the patients, the patients were classified into 5-year age groups per 100,000 persons in order to determine age- and sexadjusted incidence rates. Additional analyses on medical costs, length of hospitalization stay, visits to outpatient clinics, examinations (magnetic resonance imaging [MRI], bone scans) and medical procedures (conservative treatment, percutaneous vertebroplasty, kyphoplasty), and type of medical institute (tertiary general hospital, general hospital, hospital, clinic, others) were performed to analyze the socioeconomic costs of OVCF (Fig. 1). Medical institutes were classified by inpatient bed size: clinics $(<30$ beds), hospitals (30-99 beds), general hospitals ( $\geq 100$ beds), and tertiary hospitals (general hospitals approved to provide the most advanced medical care and to treat severely ill patients, with at least 20 departments); the "others" category included convalescent hospitals, public health centers, and health and medical centers.

\section{Healthcare cost estimation}

Healthcare costs are expenses on medical procedures and services associated with disease management performed in hospitals, clinics, and other medical institutes. These include costs for hospitalization, outpatient visits, and prescription drugs. When clinics and hospitals manage insured patients, they request medical cost reimbursement from the Korean National Health Insurance (KNHI) at the end of each month. HIRA determines the adequacy of each claim and keeps relevant data in its database. As the HIRA database covers osteoporosis and related diseases, nearly all OVCF cases are also covered. In this study, health insurance claims data were analyzed to calculate both inpatient and outpatient costs related to OVCF. Claims amounts for the first visit and followup management were tallied for each patient. Inpatient 
Table 1. ICD-10 codes for searching cohorts

\begin{tabular}{|c|c|c|c|}
\hline Parameters & Category & Codes & Description \\
\hline \multirow[t]{7}{*}{ ICD-10 code } & Osteoporosis & M80 & Osteoporosis with pathologic fracture \\
\hline & & M81 & Osteoporosis without pathologic fracture \\
\hline & & M82 & Osteoporosis in diseases classified elsewhere \\
\hline & Vertebral fracture & S220 & Fracture of thoracic vertebra \\
\hline & & S320 & Fracture of lumbar vertebra \\
\hline & & M48.4 & Fatigue fracture of vertebra \\
\hline & & M48.5 & Collapsed vertebra, not elsewhere classified \\
\hline \multirow[t]{8}{*}{ Examination code } & $\mathrm{MRl}$ & HE110 & Thoracic MRI without contrast \\
\hline & & HE210 & Thoracic MRI with contrast \\
\hline & & HE510 & Thoracic MRI with 3D reconstruction \\
\hline & & HE111 & Lumbosacral MRI without contrast \\
\hline & & HE211 & Lumbosacral MRI with contrast \\
\hline & & $\mathrm{H} 511$ & Lumbosacral MRI with 3D reconstruction \\
\hline & Bone scan & HC190 & Partial body bone scan \\
\hline & & HC191 & Whole body bone scan \\
\hline \multirow[t]{4}{*}{ Procedure code } & Vertebroplasty & N0471 & Single level vertebroplasty \\
\hline & & N0472 & Multiple level vertebroplasty \\
\hline & Kyphoplasty & N0473 & Single level kyphoplasty \\
\hline & & N0474 & Multiple level kyphoplasty \\
\hline
\end{tabular}

ICD-10, 10th revision of the International Statistical Classification of Diseases and Related Health Problems; MRI, magnetic resonance imaging; 3D, three-dimensional.

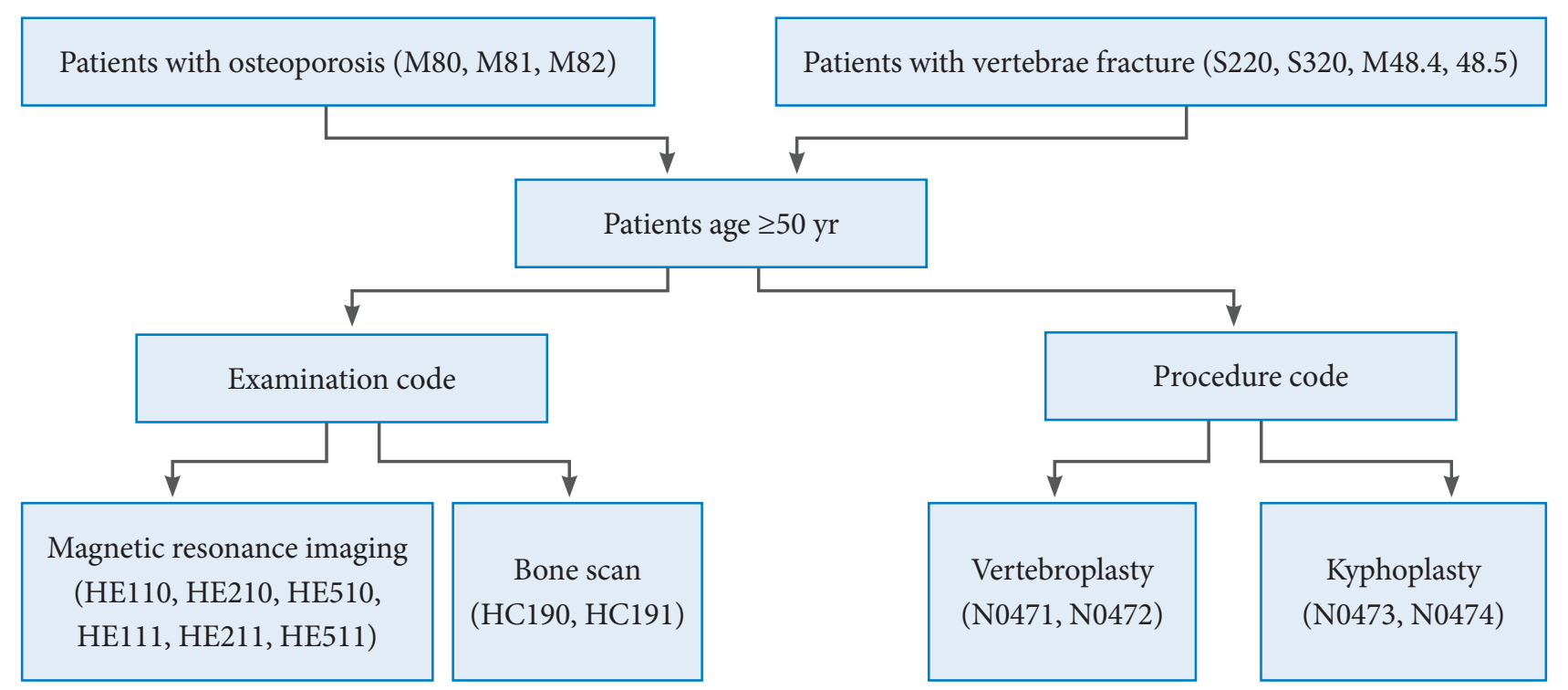

Fig. 1. Flowcharts of osteoporotic vertebral compression fracture patients data in this study.

drug expenses already included drug costs incurred during hospitalization. Outpatient drug expenses were also calculated using the HIRA database. The HIRA database is nationally representative of medical care costs covered by the KNHI program. However, the HIRA database does not include noncovered costs, such as costs of assistive devices, caregivers, and over-the-counter (OTC) drugs.

All costs were calculated in Korean won (KRW) and 
then converted to US dollars (USD) by using the 2017 year average conversion rate of $1,130 \mathrm{KRW}$ per $1 \mathrm{USD}$ (http//ecos.bok.or.kr/).

\section{Statistical analysis}

Age-adjusted and sex-specific incidence rates of OVCF were calculated per 100,000 persons. The 2014 Organization for Economic Cooperation and Development population (http://stats.oecd.org) was taken as the standard population. Estimated year-, age-, and sex- populations were obtained from the Statistics Korea website (http:// www.kosis.kr). Variation in the proportion and trend of examination or treatment over the years was analyzed using the Cochran-Armitage trend test with SAS statistical software ver. 9.13 (SAS Institute Inc., Cary, NC, USA). A $p<0.05$ was considered statistically significant.

\section{Ethics statement}

The study protocol was approved by the institutional review board of the Hanyang University Hospital (Seoul, South Korea; HYUH 2017-08-039).

\section{Results}

There were 644,500 OVCF cases from 2012 to 2016. The yearwise details were as follows (Table 2): (1) 2012: population aged $>50$ years, $15,649,477 ; 117,361(0.75 \%)$ OVCF patients (17,373 men and 99,988 women; gender ratio, 1:5.76); mean age of OVCF occurrence, 74.05 years; (2) 2013: population aged $>50$ years, $16,288,186 ; 125,253$ (0.77\%) OVCF patients (18,622 men and 106,631 women; gender ratio, 1:5.73); mean age of OVCF occurrence, 74.24 years; (3) 2014: population aged $>50$ years, $16,852,148$; 127,207 (0.75\%) OVCF patients (19,244 men and 107,963 women; gender ratio, 1:5.61); mean age of OVCF occur-

Table 2. Gender proportion of osteoporotic vertebral compression fracture patients during 5 years

\begin{tabular}{|c|c|c|c|c|c|}
\hline \multirow{2}{*}{ Variable } & \multicolumn{5}{|c|}{ Year } \\
\hline & 2012 & 2013 & 2014 & 2015 & 2016 \\
\hline Populations & $15,649,477$ & $16,288,186$ & $16,852,148$ & $17,465,255$ & $18,071,780$ \\
\hline Patients & 117,361 & 125,253 & 127,207 & 134,790 & 139,889 \\
\hline Male & 17,373 & 18,622 & 19,244 & 21,040 & 21,745 \\
\hline Female & 99,988 & 106,631 & 107,963 & 113,750 & 118,144 \\
\hline Gender ratio & 5.76 & 5.73 & 5.61 & 5.41 & 5.43 \\
\hline Mean age (yr) & 74.05 & 74.24 & 74.71 & 74.94 & 75.15 \\
\hline
\end{tabular}

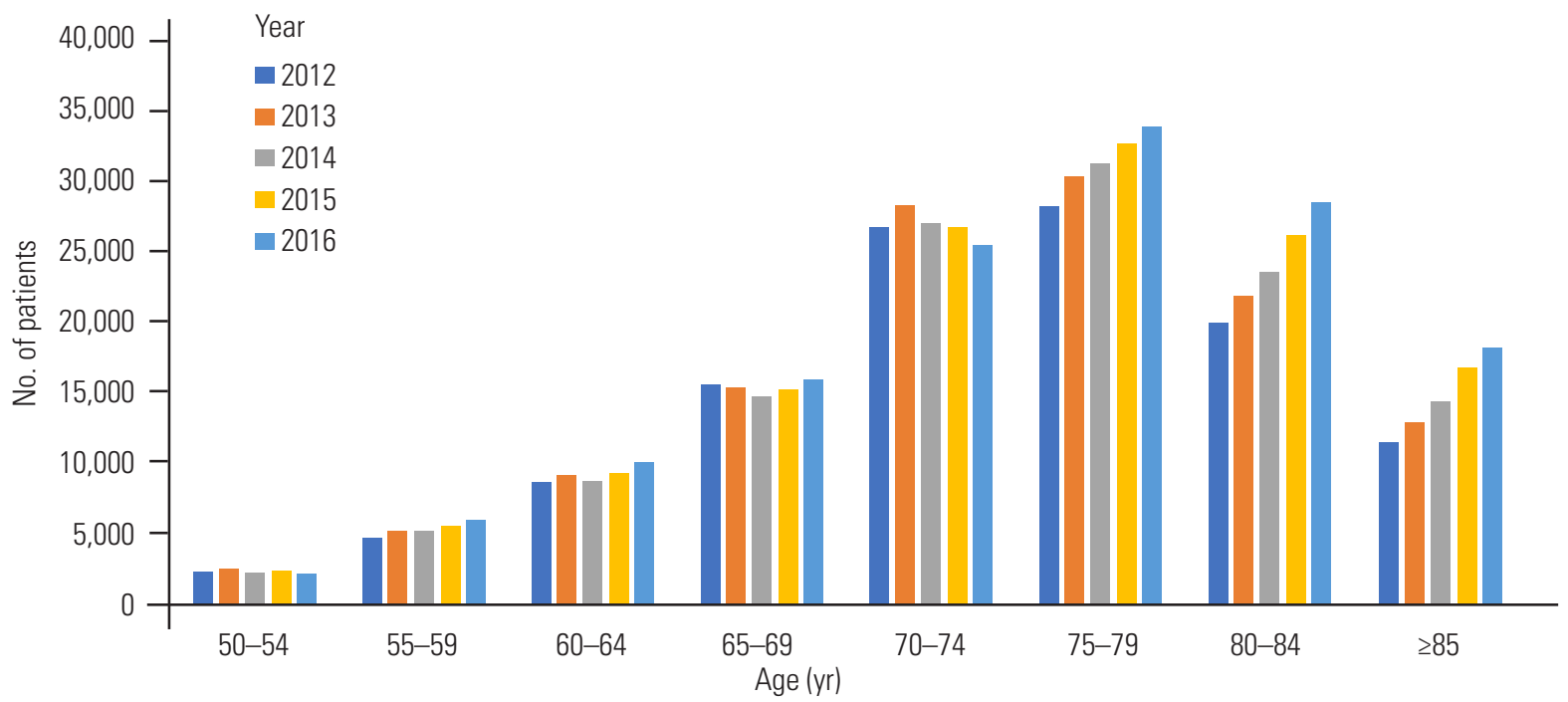

Fig. 2. Age group of osteoporotic vertebral compression fracture patients during 5 years (2012-2016). 
rence, 74.71 years; (4) 2015: population aged $>50$ years, $17,465,255 ; 134,790$ (0.77\%) OVCF patients $(21,040$ men and 113,750 women; gender ratio, 1:5.41, with the rate for women continuously decreasing); mean age of OVCF occurrence, 74.94 years; and (5) 2016: population aged $>50$ years, $18,071,780 ; 139,889$ (0.77\%) OVCF patients $(21,745$ men and 118,144 women; gender ratio, 1:5.43); mean age of OVCF occurrence, 75.15 years.

Analyzed sequentially by year, the number of OVCF patients increased from 117,361 in 2012 to 139,889 in 2016 $(p<0.001)$. Analyzed by gender, the number of men with OVCF increased from 17,373 in 2012 to 21,745 in 2016 and the number of women with OVCF also increased from 99,988 in 2012 to 118,144 in 2016.

OVCF occurred most frequently in patients aged $>70$ years (45\%) (Fig. 2). The age-adjusted incidence rate per 100,000 persons increased from 840.49 cases in 2012 to
869.68 cases in $2016(0.70 \%, p=0.20)$. The sex-adjusted incidence rate per 100,000 persons was 330.63 men and $1,185.31$ women in 2012 , which increased to 352.54 men and 1,222.14 women in 2016. Both indices increased considerably in 2016 compared to 2012, and the change in the annual mean percentage was higher in men $(1.8 \%$, $p<0.001)$ compared to women $(0.5 \%, p=0.30)$ (Table 3$)$.

From 2012 to $2016,57,451$ patients $(8.9 \%)$ visited the emergency department; of those, 31,196 (54.3\%) were hospitalized and 20,108 (35\%) underwent MRI. The visits to the emergency department, number of hospitalizations, and MRI examinations all increased from 2012 to 2016. In 2012, 63,859 (54.4\%) patients were hospitalized for an average of 13.89 days; of those, 10,474 (8.92\%) visited the emergency department. In 2016, the number of hospitalized patients increased to 76,322 (54.6\%); of those, 13,029 (9.31\%) visited the emergency department. The MRI

Table 3. Number of cases and age-adjusted rates of osteoporotic vertebral compression fracture from 2012 to 2016

\begin{tabular}{|c|c|c|c|c|c|c|c|c|c|c|c|c|}
\hline \multirow{2}{*}{ Year } & \multicolumn{6}{|c|}{ No. of cases } & \multicolumn{6}{|c|}{ Age-adjusted rates per 100,000 persons ${ }^{\text {a) }}$} \\
\hline & Total & $p$-value ${ }^{\mathrm{b})}$ & Men & $p$-value ${ }^{\mathrm{b})}$ & Women & $p$-value $\mathrm{b}^{\mathrm{b})}$ & Total & $p$-value ${ }^{\text {b) }}$ & Men & $p$-value ${ }^{\mathrm{b})}$ & Women & $p$-value ${ }^{\mathrm{b})}$ \\
\hline 2012 & 117,361 & & 17,373 & & 99,988 & & 840.49 & & 330.63 & & 1185.31 & \\
\hline 2013 & 125,253 & & 18,622 & & 106,631 & & 857.19 & & 332.94 & & 1211.62 & \\
\hline 2014 & 127,207 & & 19,244 & & 107,963 & & 837.88 & & 332.18 & & 1179.83 & \\
\hline 2015 & 134,790 & & 21,040 & & 113,750 & & 855.96 & & 350.43 & & 1199.01 & \\
\hline 2016 & 139,889 & & 21,745 & & 118,144 & & 869.68 & & 352.54 & & 1222.14 & \\
\hline Overall & 644,500 & $<0.001$ & 98,024 & $<0.001$ & 546,476 & $<0.001$ & - & 0.20 & - & $<0.001$ & - & 0.30 \\
\hline
\end{tabular}

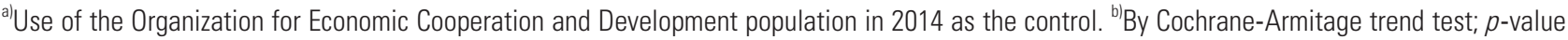
$<0.001$.

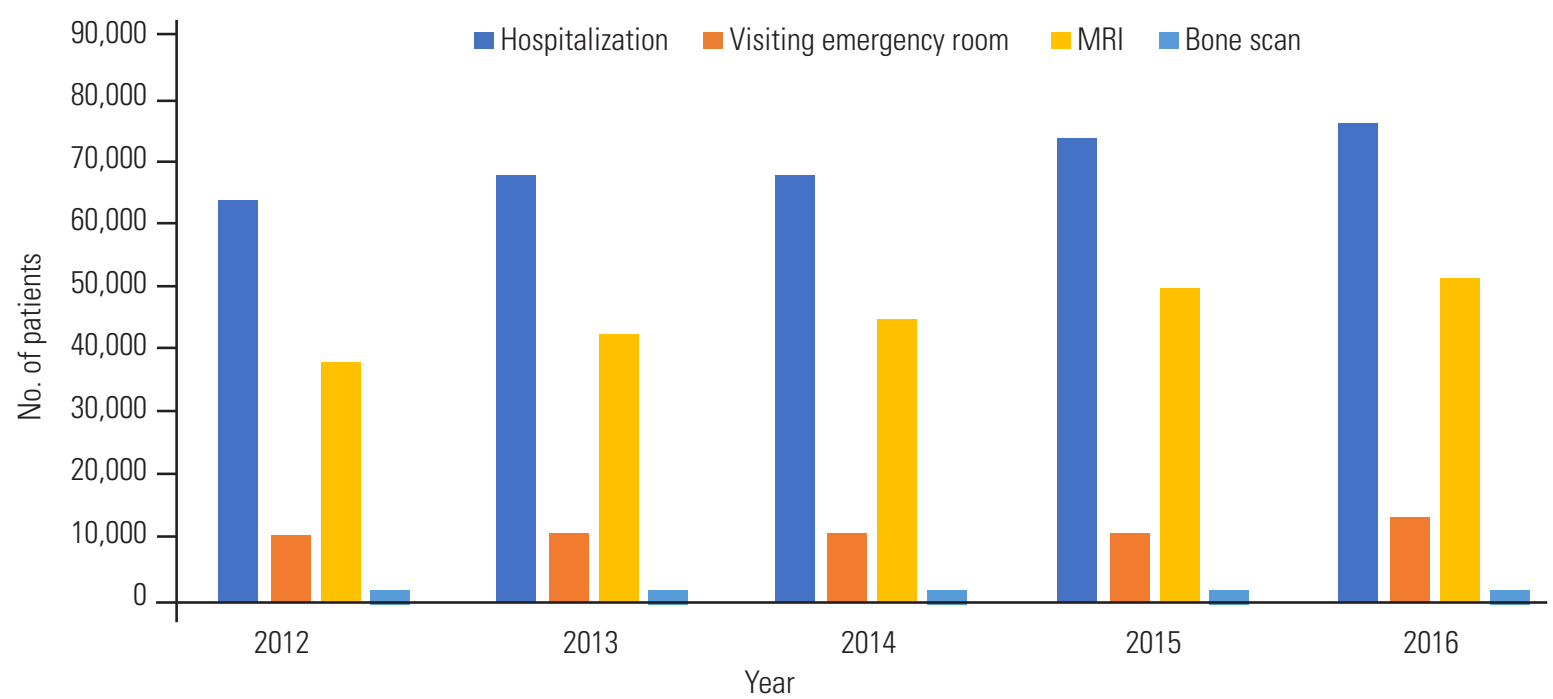

Fig. 3. Hospitalization and MRI examination data for osteoporotic vertebral compression fracture patients. MRI, magnetic resonance imaging. 
examinations increased from 38,190 (32.5\%) in 2012 to 52,506 (37.5\%) in 2016 (Fig. 3).

The rate of the bone cement augmentation increased from $23.4 \%$ (27,471 patients) in 2012 to $25.2 \%(35,311$ patients) in 2016, while the rate of conservative treatment decreased from $76.5 \%(89,890$ patients) in 2012 to $74.7 \%$ $(104,578$ patients) in $2016(p<0.001)$ (Table 4$)$. In this study, bone cement augmentation was taken to include percutaneous vertebroplasty and kyphoplasty, which increased from 38,860 (33.1\%) patients in 2012 to 48,783 (34.8\%) patients in 2016 and from 6,085 (5.18\%) patients to $7,040(5.03 \%)$ patients $(p<0.001)$, respectively. The

Table 4. Proportion of treatment modality treated with osteoporotic vertebral compression fracture

\begin{tabular}{|c|c|c|c|c|}
\hline \multirow{2}{*}{ Year } & \multicolumn{2}{|c|}{$\begin{array}{l}\text { Conservative } \\
\text { treatment }\end{array}$} & \multicolumn{2}{|c|}{$\begin{array}{l}\text { Bone cement } \\
\text { augmentation }\end{array}$} \\
\hline & Total & $p$-value ${ }^{\text {a) }}$ & Total & $p$-value ${ }^{\text {a) }}$ \\
\hline 2012 & 89,890 (76.59) & $<0.001$ & $27,471(23.41)$ & $<0.001$ \\
\hline 2013 & $95,621(76.34)$ & & $29,632(23.66)$ & \\
\hline 2014 & 97,512 (76.66) & & $29,695(23.34)$ & \\
\hline 2015 & $101,882(75.59)$ & & $32,908(24.41)$ & \\
\hline 2016 & $104,578(74.76)$ & & $35,311(25.24)$ & \\
\hline
\end{tabular}

Values are presented as number of persons (\%).

${ }^{a}$ By Cochrane-Armitage trend test; $p$-value $<0.001$. number of patients treated at clinics and other medical institutes decreased from $28,829(24.6 \%)$ in 2012 to 28,573 $(20.4 \%)$ in 2016 , while the number of patients treated at hospitals, general hospitals, and tertiary general hospitals increased from 88,532 (75.4\%) in 2012 to 105,699 (75.6\%) in 2016 (Table 5).

The total health insurance cost increased steadily over the 5 years: it was $\$ 193,210,353.55$ in 2012 , $\$ 209,908,733.00$ in $2013, \$ 225,300,588.77$ in $2014, \$ 256,449,303.66$ in 2015 , $\$ 281,968,877.65$ in 2016 , accompanied by a growth in the gross domestic product. Health insurance costs for conservative treatment increased from $\$ 111,845,252.52$ in 2012 to $\$ 167,896,842.72$ in 2016 , while health insurance costs for bone cement augmentation increased from \$81,365,101.03 in 2012 to $\$ 117,072,034.92$ in 2016 (Table 6).

\section{Discussion}

Many studies on osteoporosis have been conducted in South Korea. This study analyzed the age- and sex-adjusted incidence rates, management trends, and medical costs of OVCF using HIRA data. From 2012 to 2016, OVCF was most common in patients in their seventies (45\%). The mean age of OVCF occurrence was 75 years (75.15 years). The age-adjusted incidence rate per 100,000 persons was 840.49 in 2012 and 869.68 in 2016, while the sex-adjusted

Table 5. Proportion of medical institute type treated with osteoporotic vertebral compression fracture

\begin{tabular}{lrrrrr} 
& \multicolumn{3}{c}{ Year } & & \\
Variable & 2012 & 2013 & 2014 & 2015 & 2016 \\
\cline { 2 - 6 } & 8,637 & 8,845 & 9,381 & 10,014 & 10,665 \\
Tertiary general hospital & 35,505 & 39,016 & 42,047 & 45,862 & 49,345 \\
General hospital & 44,390 & 47,879 & 47,378 & 49,823 & 51,306 \\
Hospital & 25,680 & 25,930 & 24,230 & 24,244 & 23,362 \\
Clinic & 3,149 & 3,583 & 4,171 & 4,847 & 5,211 \\
\hline Others & & &
\end{tabular}

Table 6. Socio-economic costs used in the treatment of osteoporotic vertebral compression fracture

\begin{tabular}{lrrrrr} 
& \multicolumn{4}{c}{ Year } \\
Variable & \multicolumn{4}{c}{2014} & 2015 \\
\cline { 2 - 6 } & 2012 & 2013 & $1,411.33$ & $1,382.76$ & $1,411.04$ \\
Gross domestic product (US\$, billion) & $1,222.81$ & $1,305.61$ & 2016 \\
Health care cost (US\$) & $193,210,353.55$ & $209,908,733.00$ & $225,300,588.77$ & $256,449,303.66$ & $284,968,877.65$ \\
\hline Cement augmentation (US\$) & $81,365,101.03$ & $86,480,584.49$ & $88,239,650.63$ & $103,243,812.65$ & $117,072,034.92$ \\
\hline Conservative treatment (US\$) & $111,845,252.52$ & $123,428,148.51$ & $137,060,938.14$ & $153,205,491.01$ & $167,896,842.72$ \\
\hline
\end{tabular}


incidence rate per 100,000 persons was 360.63 men and 1,185.31 women in 2012 compared to 352.54 men and $1,222.14$ women in 2016. Both indices were considerably higher in 2016 compared to 2012, as also reported by Kim et al. [9], who analyzed the HIRA data for 2008-2011. They found that the age-adjusted incidence rate increased continuously from 1,127 per 100,000 persons in 2008 to 1,295 per 100,000 persons in 2011 and was higher in women.

South Korea has environmental, social, and policy factors that other countries do not have, which explains the increase in the annual OVCF incidence rate, the steady increase in the OVCF incidence rate in women, and the higher increase in the OVCF incidence rate in men. First, the elderly population in South Korea has a sedentary lifestyle, unlike Western countries, which explains the higher annual OVCF incidence rate in South Korea compared to other countries. Studies have shown the relationship between a sedentary lifestyle and osteoporotic fractures $[10,11]$. In addition, in a sedentary lifestyle, the vulnerability of the elderly to minor energy trauma increases when sitting or standing, which leads to a high OVCF incidence rate. The second reason is the change in the family size. The nuclear family ratio in South Korea is higher compared to neighboring Asian countries, such as Japan and China. The proportion of elderly people living alone has also increased recently [12]. This had led to the increased incidence of OVCF because of insufficient care at home.

Third, the steady increase in the OVCF incidence rate in women is likely associated with osteoporosis. The BMD of women peaks in their thirties and declines rapidly postmenopause [13], so women are at a nonmodifiable major risk of osteoporotic fractures [14]. In addition, the National Health Insurance Service (NHIS), the claim system for medial healthcare costs in South Korea, has set age criteria for the BMD. The criteria are applied differently by gender. The criterion for men is age $>70$ years, while the criterion for women is age $>65$ years. This difference, in turn, leads to a difference in the incidence of osteoporosis and OVCF in men and women. Higher OVCF incidence rates in women are also found in Vietnam and Taiwan but not in Japan, Thailand, Indonesia, and Hong Kong, where the OVCF incidence rate is higher in men [3]. The higher OVCF incidence rate in women is also consistent with results of research on data from China from 2003 to 2015 [15], and BMD decline in South Korean women is found to be significantly faster compared to Caucasian or Lebanese women [16]. In addition, differences in the rate of BMD decline seem to be more heavily affected by environmental differences compared to race [3].

Finally, the reason the OVCF incidence rate is higher in men than in women is related to a social interest in osteoporosis. The NHIS system BMD age criteria were announced in 2007. Since then, the social interest in osteoporosis has increased, and so has the frequency of osteoporosis tests in men. As a result, the diagnosis of osteoporosis in men has increased, which can be attributed to an increase in OVCF.

The duration of treatment decreased slightly from 13.89 days in 2012 to 13.27 days in 2016, but the number of hospitalizations, visits to the emergency department, and MRI examinations consistently increased. In addition, the number of patients treated at clinics and other medical institutes decreased slightly, but the number of patients treated at hospitals, general hospitals, and tertiary general hospitals increased from 88,532 patients in 2012 to 105,699 patients in 2016, which increase appears to have played a role in increasing the total health insurance cost for OVCF. The gradual increase in the number of patients undergoing treatment at higher-level hospitals was also reported by Jang et al. [17], who analyzed the 2007 data of HIRA.

The total health insurance cost increased from $\$ 193,210$, 353.55 in 2012 to $\$ 284,968,877.65$ in 2016. Although the treatment duration slightly decreased, the total health insurance cost significantly increased, probably because of the increased MRI examinations for OVCF. In terms of management trends, the rate of bone cement augmentation increased, while the rate of conservative treatment decreased. These findings showed an upward trend, which was seen in the NHIS data for 2008 to 2011 [9]. According to the NHIS data for 2008-2011, the total healthcare costs of osteoporotic fractures increased from $\$ 549$ million in 2008 to $\$ 722$ million in 2011 . The most expensive fracture for both genders was spine fracture, accounting for $43 \%-45 \%$ of the total healthcare costs. There was a similar trend in the US data for 2005 published by Burge et al. [6]. On the basis of these results, the relative scale of OVCF costs can be predicted, which emphasizes the need for prevention and management strategies for OVCF. In addition, to decrease the economic burden of OVCF, government officials and health authorities can make informed decisions about various strategies. The following measures can be 
taken: appropriate education for osteoporosis medication, periodic monitoring of osteoporosis by various test modalities, and customized management for probable OVCF patients according to the risk of falling down. Kim et al. [18] developed a prediction model for the risk of osteoporotic fractures that does not require BMD examination.

This study had several limitations. First, in the process of selecting HIRA data using ICD-10 codes, some trauma or pathological fractures might have been included. Second, only the healthcare costs covered by insurance were assessed. We extracted only total healthcare costs and did not separate them into the cost for each medical procedure and costs by age group. Considering that many elderly OVCF patients in South Korea use herbal medicines for musculoskeletal symptoms, the potential costs of OVCF could be substantially greater than shown in this study. However, nonmedical costs (e.g., OTC drugs or herbal medicines), caregiver costs, and indirect costs (e.g., lost productivity) were not evaluated, and we did not find out whether medical resources are overused. Further studies that include nonmedical and indirect costs might be necessary to evaluate the actual economic burden of OVCF in South Korea. Third, we excluded OVCF patients who underwent spinal fusion surgery instead of conservative treatment or bone cement augmentation. Fourth, as in other studies that used similar methods [17], diagnoses might have been omitted if they failed to match an appropriate code, secondary osteoporosis might have occurred, or the results of BMD were not recorded, which might have affected the OVCF incidence rate analysis.

In this study, the 5-year average OVCF incidence rate per 100,000 persons in South Korea was 852.24. It is expected that the socioeconomic costs of OVCF will gradually increase. Moreover, the total costs of OVCF are increasing because of the constant increase in the use of bone cement augmentation, as seen in US studies [6]. This study was significant in that it confirmed the OVCF management trends and high rates of occurrence reported by others.

\section{Conclusions}

Considering the results of this study and the rapid aging in the South Korean population, OVCF will be an ongoing major health problem in the elderly. Therefore, a public health strategy to minimize the economic burden of OVCF would be prudent.

\section{Conflict of Interest}

No potential conflict of interest relevant to this article was reported.

\section{ORCID}

Sung Hoon Choi: https://orcid.org/0000-0003-3328-2043

Dong-Yun Kim: https://orcid.org/0000-0002-9147-9971

Ja Wook Koo: https://orcid.org/0000-0001-5604-8271

Seung Gun Lee: https://orcid.org/0000-0001-7401-7620

Soo-Young Jeong: https://orcid.org/0000-0002-4513-0461

Chang-Nam Kang: https://orcid.org/0000-0002-4633-3391

\section{References}

1. Gallagher J, Riggs B, Eisman J. Diagnosis, prophylaxis, and treatment of osteoporosis. Am J Med 1994;90:646-50.

2. Kim SS, Lee DH, Kim JH, et al. Risk factors for subsequent vertebral compression fracture following osteoporotic compression fracture. J Korean Orthop Assoc 2016;51:479-85.

3. Ballane G, Cauley JA, Luckey MM, El-Hajj Fuleihan G. Worldwide prevalence and incidence of osteoporotic vertebral fractures. Osteoporos Int 2017;28:1531-42.

4. Sakuma M, Endo N, Oinuma T, et al. Incidence of osteoporotic fractures in Sado, Japan in 2010. J Bone Miner Metab 2014;32:200-5.

5. European Prospective Osteoporosis Study (EPOS) Group, Felsenberg D, Silman AJ, et al. Incidence of vertebral fracture in Europe: results from the European Prospective Osteoporosis Study (EPOS). J Bone Miner Res 2002;17:716-24.

6. Burge R, Dawson-Hughes B, Solomon DH, Wong JB, King A, Tosteson A. Incidence and economic burden of osteoporosis-related fractures in the United States, 2005-2025. J Bone Miner Res 2007;22:465-75.

7. Boschitsch EP, Durchschlag E, Dimai HP. Age-related prevalence of osteoporosis and fragility fractures: real-world data from an Austrian menopause and osteoporosis clinic. Climacteric 2017;20:157-63.

8. Johnell O, Kanis JA, Oden A, et al. Mortality after osteoporotic fractures. Osteoporos Int 2004;15:38-42.

9. Kim HY, Ha YC, Kim TY, et al. Healthcare costs of osteoporotic fracture in Korea: information from the 
National Health Insurance claims database, 20082011. J Bone Metab 2017;24:125-33.

10. McGraw RL, Riggs JE. Osteoporosis, sedentary lifestyle, and increasing hip fractures: pathogenic relationship or differential survival bias. Calcif Tissue Int 1994;55:87-9.

11. Lousuebsakul-Matthews V, Thorpe D, Knutsen R, Beeson WL, Fraser GE, Knutsen SF. Non-sedentary lifestyle can reduce hip fracture risk among older Caucasians adults: the Adventist Health Study-2. Br J Med Med Res 2015;8:220-9.

12. Korean Statistical Information Service. Summary of census population [Internet]. Daejeon: Statistics Korea; 2018 [cited 2018 Mar 7]. Available from: http:// kostat.go.kr/portal/korea/index.action.

13. Sorenson JA, Cameron JR. A reliable in vivo measurement of bone-mineral content. J Bone Joint Surg Am 1967;49:481-97.
14. Shin CS, Kim MJ, Shim SM, et al. The prevalence and risk factors of vertebral fractures in Korea. J Bone Miner Metab 2012;30:183-92.

15. Chen P, Li Z, Hu Y. Prevalence of osteoporosis in China: a meta-analysis and systematic review. BMC Public Health 2016;16:1039.

16. Cui LH, Choi JS, Shin MH, et al. Prevalence of osteoporosis and reference data for lumbar spine and hip bone mineral density in a Korean population. J Bone Miner Metab 2008;26:609-17.

17. Jang S, Park C, Jang S, et al. Medical service utilization with osteoporosis. Endocrinol Metab 2010;25:326-39.

18. Kim HY, Jang EJ, Park B, et al. Development of a Korean Fracture Risk Score (KFRS) for predicting osteoporotic fracture risk: analysis of data from the Korean National Health Insurance Service. PLoS One 2016;11:e0158918. 